\title{
Error Estimates for the Difference Method to System of Ordinary Differential Equations with Boundary Layer
}

\author{
Ilhame Amirali \\ Department of Mathematics, Faculty of Art and Science, Sinop University, Sinop, Turkey \\ Email: ailhame@gmail.com
}

Received August 14, 2013; revised September 15, 2013; accepted October 1, 2013

Copyright (C) 2013 Ilhame Amirali. This is an open access article distributed under the Creative Commons Attribution License, which permits unrestricted use, distribution, and reproduction in any medium, provided the original work is properly cited.

\begin{abstract}
This work deals with the numerical solution of singular perturbation system of ordinary differential equations with boundary layer. For the numerical solution of this problem fitted finite difference scheme on a uniform mesh is constructed and analyzed. The uniform error estimates for the approximate solution are obtained.
\end{abstract}

Keywords: Singular Perturbation; Linear System; Difference Scheme; Uniform Convergence; Error Estimates

\section{Introduction}

We consider the initial-boundary value problem for the linear system of ordinary differential equations in the interval $[0,1]$ :

$$
\begin{aligned}
& L_{1} u:=\varepsilon u^{\prime \prime}+a_{1}(x) u^{\prime}+b_{1}(x) u \\
&= c_{1}(x) v+f_{1}(x), \quad 0<x<1, \\
& L_{2} v:=\varepsilon v^{\prime \prime}+a_{2}(x) v^{\prime}-b_{2}(x) v \\
&= c_{2}(x) u+f_{2}(x), \quad 0<x<1, \\
& u(0)=A_{1}, u^{\prime}(0)=\frac{B_{1}}{\varepsilon}, \\
& v^{\prime}(0)=\frac{A_{2}}{\varepsilon}, \quad v(1)=B_{2} .
\end{aligned}
$$

where $\varepsilon$ is a small parameter, $A_{1}, A_{2}, B_{1}, B_{2}$ are given constants. The functions $a_{i}(x) \geq \alpha_{i}>0, \quad b_{i}(x)$, $c_{i}(x), f_{i}(x)(i=1,2)$ are given functions satisfying certain regularity conditions will be specified whenever necessarily.

The above type initial/boundary value problems arise in many areas of mechanics and physics [1,2].

Differential equations with a small parameter $\varepsilon$ multiplying the highest-order derivative terms are said to be singularly perturbed. They occur frequently in mathematical problems in the sciences and engineering for example, in fluid flow at high Reynold number, electrical networks, chemical reactions, control theory, the equations governing flow in porous media, the drift-diffusion equations of semi-conductor device physics, and other physical models. The mathematical models describing these phenomena contain a small parameter $\varepsilon$ and the influence of this parameter reveal itself in a sudden change of the dependent variable $u_{\varepsilon}$ taking place with in a small layer. That is, the solution of this type of problem has a narrow region in which the solution changes rapidly and the outside solution changes smoothly [1-3].

It is well-known that standard discretization methods do not work well for these problems as they often produce oscillatory solutions which are inaccurate if the perturbed parameter $\varepsilon$ is small. To obtain robust numerical methods, it is necessary to fix the coefficients (fitted operator methods) or the mesh (fitted mesh methods) to the behavior of the exact solution [2,4].

In this present paper, we analyze the numerical solution of the initial/boundary problem (1)-(4). The numerical method presented here comprises a fitted difference scheme on a uniform mesh. Fitted operator method is widely used to construct and analyse uniform difference methods, especially for a linear differential problems (see, e.g., [4-7]). In the Section 2, we state some important properties of the exact solution. The derivations of the difference scheme and uniform convergence analysis have been given in Section 3. Uniform convergence is proved in the discrete maximum norm. The approach to the construction of the discrete problem and the error 
analysis for the approximate solution are similar to those in $[8,9]$.

Difference schemes for singularly perturbed systems with another type of initial/boundary conditions were investigated in [3,6,9-11].

Throughout the paper, $C$ will denote a generic positive constant independent of $\varepsilon$ and of the mesh parameter.

\section{Analytical Results}

Lemma 2.1. Under the

$$
\rho=\left(\alpha_{1} \alpha_{2}\right)^{-1}\left\|c_{1}\right\|_{\infty}\left\|c_{2}\right\|_{\infty} \exp \left(\alpha_{1}\left\|b_{1}\right\|_{\infty}\right)<1
$$

the solution of the problem (1)-(4) satisfies

$$
\begin{gathered}
\|u\|_{\infty} \leq C, \\
\left|u^{\prime}(x)\right| \leq C\left(1+\frac{1}{\varepsilon} \exp \left(\frac{-\alpha_{1} x}{\varepsilon}\right)\right), \\
\|v\|_{\infty} \leq C, \\
\left|v^{\prime}(x)\right| \leq C\left(1+\frac{1}{\varepsilon} \exp \left(\frac{-\alpha_{2} x}{\varepsilon}\right)\right) .
\end{gathered}
$$

Here $\|g\|_{\infty}=\max _{[0,1]}|g(x)|$ for any continuous functions $g(x)$.

Proof. First we prove that for the solution of initial-value problem of the type

$$
F_{1}(x)=c_{1}(x) v(x)+f_{1}(x)
$$

the following estimates hold

$$
\begin{aligned}
\|u\|_{\infty} \leq & \exp \left(\left\|b_{1}\right\|_{\infty} \alpha_{1}^{-1}\right)\left(\left|A_{1}\right|+\alpha_{1}^{-1}\left|B_{1}\right|\right. \\
& \left.+\alpha_{1}^{-1}\|v\|_{\infty}\left\|c_{1}\right\|_{\infty}+\alpha_{1}^{-1}\left\|f_{1}\right\|_{\infty}\right),
\end{aligned}
$$

$$
\begin{aligned}
& \left|u^{\prime}(x)\right| \\
& \leq \exp \left(\left\|b_{1}\right\|_{\infty} \alpha_{1}^{-1}\right)\left\|b_{1}\right\|_{\infty}\left|A_{1}\right|+\left\|b_{1}\right\|_{\infty} \alpha_{1}^{-1} \exp \left(\left\|b_{1}\right\|_{\infty} \alpha_{1}^{-1}\right)\left|B_{1}\right| \\
& +\frac{1}{\varepsilon}\left(\frac{\alpha_{1}^{-1} x}{\varepsilon}\left|B_{1}\right|+\alpha_{1}^{-1}\left(\left\|b_{1}\right\|_{\infty} \exp \left(\left\|b_{1}\right\|_{\infty} \alpha_{1}^{-1}\right)+1\right)\left\|c_{1}\right\|_{\infty}\|v\|_{\infty}\right. \\
& \left.\quad+\alpha_{1}^{-1}\left(\left\|b_{1}\right\|_{\infty} \exp \left(\left\|b_{1}\right\|_{\infty} \alpha_{1}^{-1}\right)+1\right)\left\|f_{1}\right\|_{\infty}\right)
\end{aligned}
$$

Using the function $F_{2}(x)=c_{2}(x) u(x)+f_{2}(x)$, to prove (10) after some manipulations we have

$$
\|v\|_{\infty} \leq\left|A_{2}\right| \alpha_{2}^{-1}+\alpha_{2}^{-1}\left\|c_{2}\right\|_{\infty}\|u\|_{\infty}+\alpha_{2}^{-1}\left\|f_{2}\right\|_{\infty}+\left|B_{2}\right|,
$$

$$
\begin{aligned}
& \left|v^{\prime}(x)\right| \leq \frac{\left|A_{2}\right|}{\varepsilon} \exp \left(\frac{-\alpha_{2} x}{\varepsilon}\right)+\alpha_{2}^{-1}+\alpha_{2}^{-1}\left(\left\|b_{2}\right\|_{\infty} \alpha_{2}^{-1}+1\right) \\
& \times\left(\left\|c_{2}\right\|_{\infty}\|u\|_{\infty}+\left\|f_{2}\right\|_{\infty}\right)+\alpha_{2}^{-1}\left\|b_{2}\right\|_{\infty}\left(\alpha_{2}^{-1}\left|A_{2}\right|+\left|B_{2}\right|\right) .
\end{aligned}
$$

\section{Hence}

$$
\begin{aligned}
\|u\|_{\infty} \leq & (1-p)^{-1}\left(\exp \left(\left\|b_{1}\right\|_{\infty} \alpha_{1}^{-1}\right)\left|A_{1}\right|\right. \\
& +\alpha_{1}^{-1} \exp \left(\left\|b_{1}\right\|_{\infty} \alpha_{1}^{-1}\right)\left|B_{1}\right| \\
& +\left(\alpha_{1} \alpha_{2}\right)^{-1}\left\|c_{1}\right\|_{\infty}\left|A_{2}\right| \exp \left(\left\|b_{1}\right\|_{\infty} \alpha_{1}^{-1}\right) \\
& +\left(\alpha_{1} \alpha_{2}\right)^{-1}\left\|c_{1}\right\|_{\infty} \exp \left(\left\|b_{1}\right\|_{\infty} \alpha_{1}^{-1}\right)\left\|f_{2}\right\|_{\infty} \\
& \left.+\exp \left(\left\|b_{1}\right\|_{\infty} \alpha_{1}^{-1}\right)\left|B_{1}\right|+\alpha_{1}^{-1} \exp \left(\left\|b_{1}\right\|_{\infty} \alpha_{1}^{-1}\right)\left\|f_{1}\right\|_{\infty}\right)
\end{aligned}
$$

and

$$
\|v\|_{\infty} \leq(1-p)^{-1}\left(\left|A_{2}\right| \alpha_{2}^{-1}+\alpha_{2}^{-1}\left\|c_{2}\right\|_{\infty}\left|A_{1}\right| \exp \left(\left\|b_{1}\right\|_{\infty} \alpha_{1}^{-1}\right)\right.
$$$$
+\alpha_{2}^{-1} \exp \left(\left\|b_{1}\right\|_{\infty} \alpha_{1}^{-1}\right)\left\|c_{2}\right\|_{\infty}\left|B_{1}\right|
$$$$
\left.+\left(\alpha_{1} \alpha_{2}\right)^{-1}\left\|c_{2}\right\|_{\infty} \exp \left(\left\|b_{1}\right\|_{\infty} \alpha_{1}^{-1}\right)\left\|f_{1}\right\|_{\infty}+\alpha_{2}^{-1}\left\|f_{2}\right\|_{\infty}+\left|B_{2}\right|\right) .
$$

Then from (9)-(12) the following estimates hold

$$
\begin{aligned}
\left|u^{\prime}(x)\right| \leq & \exp \left(\left\|b_{1}\right\|_{\infty} \alpha_{1}^{-1}\right)\left\|b_{1}\right\|_{\infty}\left|A_{1}\right|+\left\|b_{1}\right\|_{\infty} \alpha_{1}^{-1}\left|B_{1}\right| \exp \left(\left\|b_{1}\right\|_{\infty} \alpha_{1}^{-1}\right)+\left(\exp \left(\frac{-\alpha_{1} x}{\varepsilon}\right) / \varepsilon\right)\left|B_{1}\right| \\
& +\alpha_{1}^{-1}\left(\left\|b_{1}\right\|_{\infty} \exp \left(\left\|b_{1}\right\|_{\infty} \alpha_{1}^{-1}\right)+1\right)\left\|c_{1}\right\|_{\infty}(1-p)^{-1}\left(\left|A_{2}\right| \alpha_{2}^{-1}+\alpha_{2}^{-1}\left\|c_{2}\right\|_{\infty}\left|A_{1}\right| \exp \left(\left\|b_{1}\right\|_{\infty} \alpha_{1}^{-1}\right)+\alpha_{2}^{-1}\left\|c_{2}\right\|_{\infty}\left|B_{1}\right|\right. \\
& \left.\times \exp \left(\left\|b_{1}\right\|_{\infty} \alpha_{1}^{-1}\right)+\left(\alpha_{1} \alpha_{2}\right)^{-1}\left\|c_{2}\right\|_{\infty} \exp \left(\left\|b_{1}\right\|_{\infty} \alpha_{1}^{-1}\right)\left\|f_{1}\right\|_{\infty}+\alpha_{2}^{-1}\left\|f_{2}\right\|_{\infty}+\left|B_{2}\right|\right)+\left(\left\|b_{1}\right\|_{\infty} \exp \left(\left\|b_{1}\right\|_{\infty} \alpha_{1}^{-1}\right)+1\right)\left\|f_{1}\right\|_{\infty}, \\
\left|v^{\prime}(x)\right| \leq & \frac{\left|A_{2}\right|}{\varepsilon} \exp \left(\frac{-\alpha_{1} x}{\varepsilon}\right)+\alpha_{2}^{-1}\left(\left\|b_{2}\right\|_{\infty} \exp \left(\left\|b_{1}\right\|_{\infty} \alpha_{2}^{-1}\right)+1\right)\left(\left\|c_{1}\right\|_{\infty}(1-p)^{-1}\right) \exp \left(\left\|b_{1}\right\|_{\infty} \alpha_{1}^{-1}\right)\left|A_{1}\right| \\
& +\alpha_{1}^{-1} \exp \left(\left\|b_{1}\right\|_{\infty} \alpha_{1}^{-1}\right)\left|B_{1}\right|+\left(\alpha_{1} \alpha_{2}\right)^{-1}\left\|c_{1}\right\|_{\infty}\left|A_{2}\right| \exp \left(\left\|b_{1}\right\|_{\infty} \alpha_{1}^{-1}\right)+\left(\alpha_{1} \alpha_{2}\right)^{-1}\left\|c_{1}\right\|_{\infty}\left\|f_{1}\right\|_{\infty} \exp \left(\left\|b_{1}\right\|_{\infty} \alpha_{1}^{-1}\right) \\
& \left.+\exp \left(\left\|b_{1}\right\|_{\infty} \alpha_{1}^{-1}\right) \alpha_{1}\left\|c_{1}\right\|_{\infty}\left|B_{2}\right|+\alpha_{1}^{-1} \exp \left(\left\|b_{1}\right\|_{\infty} \alpha_{1}^{-1}\right)\left\|f_{1}\right\|_{\infty}\right)+\left\|f_{2}\right\|_{\infty}+\alpha_{2}^{-1}\left\|c_{2}\right\|_{\infty}\left|B_{1}\right| \exp \left(\left\|b_{1}\right\|_{\infty} \alpha_{1}^{-1}\right) \\
& +\left(\alpha_{1} \alpha_{2}\right)^{-1}\left\|c_{2}\right\|_{\infty} \exp \left(\left\|b_{1}\right\|_{\infty} \alpha_{1}^{-1}\right)\left\|f_{1}\right\|_{\infty}+\left\|f_{2}\right\|_{\infty}+\alpha_{2}^{-1}\left\|b_{2}\right\|_{\infty}\left(\alpha_{2}^{-1}\left|A_{2}\right|+\left|B_{2}\right|\right) .
\end{aligned}
$$


The last inequalities show the validity of (5)-(8).

\section{The Difference Scheme and Convergence}

Now we construct the difference scheme and investigate it. In what follow, we denote by $\omega$ the uniform mesh in $[0,1]$ :

$$
\omega_{h}=\left\{x_{i}=i h, i=1,2, \cdots, N-1, h=1 / N\right\}
$$

And $\bar{\omega}=\omega \bigcup\{x=0, l\}$. Before describing our numerical method, we introduce some notation for the mesh functions. For any mesh function $g(x)$, we use

$$
\begin{aligned}
& g_{i}=g\left(x_{i}\right), \\
& g_{\bar{x}, i}=\frac{g_{i}-g_{i-1}}{h}, \quad g_{x, i}=\frac{g_{i+1}-g_{i}}{h}, \\
& g_{x, i}=\frac{g_{i+1}-g_{i-1}}{2 h}, \quad g_{\bar{x} x, i}=\frac{g_{i+1}-2 g_{i}+g_{i-1}}{h^{2}}, \\
& \|g\|_{\infty} \equiv\|g\|_{\infty, d}=\max _{o, i, N}\left|g_{i}\right| .
\end{aligned}
$$

On $\bar{\omega}$ we propose the following difference scheme for approximating (1)-(4):

$$
\begin{gathered}
L_{h}^{1} U_{i} \equiv \varepsilon \sigma_{i}^{(1)} U_{\bar{x} x, i}+a_{1}^{i} U_{o, i}+b_{1}^{i} U_{i} \\
=c_{1}^{i} V_{i}+f_{1}^{i}, i=1,2, \cdots, N-1, \\
L_{h}^{2} V_{i} \equiv \varepsilon \sigma_{i}^{(2)} V_{\bar{x} x, i}+a_{2}^{i} V_{o}+b_{2}^{i} V_{i} \\
=c_{2}^{i} U_{i}+f_{2}^{i}, i=1,2, \cdots, N-1, \\
U_{o}=A_{1}, \quad\left(1+a_{1}(0) \frac{h}{\varepsilon} \sigma_{10}^{(2)}\right)^{-1}\left(\varepsilon^{-1} B_{1}-b_{1}(0) \frac{h}{\varepsilon} \sigma_{10}^{(2)}\right. \\
\left.U_{x, o}=(10)\right), \\
\left.+\frac{h}{\varepsilon} \sigma_{10}^{(2)} f_{1}(0)\right) \\
V_{x, 0}=\frac{A_{2}\left(1-\exp \left(-a_{2}(0) \frac{h}{\varepsilon}\right)\right)}{\alpha_{2}(0) h}, V_{N}=B_{2}
\end{gathered}
$$

where

$$
\sigma_{i}^{(k)}=\frac{h}{2 \varepsilon} a_{k}\left(x_{i}\right) \operatorname{coth} a_{k}\left(x_{i}\right) \frac{h}{2 \varepsilon},(k=1,2) .
$$

Note that

$$
\sigma_{i}^{(k)}=\frac{a_{k} h}{2 \varepsilon}\left(\sigma_{k i}^{(2)}-\sigma_{k i}^{(2)}\right)+1,
$$

where

$$
\sigma_{k i}^{(1)}=\frac{\varepsilon}{a_{k}\left(x_{i}\right) h}-\left(\exp \left(a_{k}\left(x_{i}\right) \frac{h}{\varepsilon}\right)-1\right)^{-1},
$$

$$
\sigma_{k i}^{(2)}=\left(1-\exp \left(-a_{k}\left(x_{i}\right) \frac{h}{\varepsilon}\right)\right)^{-1}-\frac{\varepsilon}{a_{k}\left(x_{i}\right) h},(k=1,2) .
$$

Throughout the paper, we assume that

$$
\rho=4 \alpha_{2}^{-1} \gamma\left\|c_{1}\right\|_{\infty}\left\|c_{2}\right\|_{\infty}<1, \gamma=4 \alpha_{1}^{-1} \exp \left(\alpha_{1}^{-1} b_{1}^{*}\right) \text {. }
$$

For solving of the (13)-(16), we giving the following iterative procedure:

$$
\begin{gathered}
L_{h}^{1} U_{i}^{(n)}=c_{1}^{i} V_{i}^{(n-1)}+f_{1}^{i}, \\
L_{h}^{2} V_{i}^{(n)}=c_{2}^{i} U_{i}^{(n)}+f_{2}^{i} .
\end{gathered}
$$

$$
\begin{aligned}
U_{0}^{(n)}= & A_{1}, \\
U_{x, 0}^{(n)}= & \left(1+\frac{a_{1} h}{\varepsilon} \sigma_{20}^{(2)}\right)^{-1}\left(\varepsilon^{-1} B_{1}-b_{1}(0) A_{1} \frac{h}{\varepsilon} \sigma_{10}^{(2)}\right. \\
& \left.+\frac{h}{\varepsilon} \sigma_{10}^{(2)} f_{1}(0)\right) \\
V_{x, 0}= & \frac{A_{2}\left(1-\exp \left(-a_{2}(0) h / \varepsilon\right)\right)}{a_{2}(0) h}, V_{N}^{(n)}=B_{2},
\end{aligned}
$$

where $V_{i}^{(0)}$ is arbitrary.

Lemma 2.2. The iteration (18)-(21) is suitable for the solution of the problem (13)-(16) and the solution of the difference problem (13)-(16) satisfies

$$
\begin{gathered}
\left\|U^{(n)}-U\right\|_{\infty} \leq \frac{\rho_{1} \rho^{n-1}}{1-\rho}\left\|V^{(1)}-V^{(0)}\right\|_{\infty}, \\
\left\|V^{(n)}-V\right\|_{\infty} \leq \frac{\rho^{n-1}}{1-\rho}\left\|V^{(1)}-V^{(0)}\right\|_{\infty} .
\end{gathered}
$$

Proof. Denoting

$$
\delta_{i}^{(n)}=U_{i}^{(n)}-U_{i-1}^{(n-1)}, \theta_{i}^{(n)}=V_{i}^{(n)}-V_{i-1}^{(n-1)}
$$

we will have

$$
\begin{gathered}
L_{1}^{h} \delta_{i}^{(n)}=c_{1}^{i} \theta_{i}^{(n-1)}, n=2, \cdots, N \\
L_{1}^{h} \theta_{i}^{(n)}=c_{2}^{i} \delta_{i}^{(n)}, \\
\delta_{0}^{(n)}=\delta_{x, 0}^{(n)}=0, \\
\theta_{x, 0}^{(n)}=0, \theta_{N}^{(n)}=0
\end{gathered}
$$

From (23)-(26) and $R_{i}=c_{1}^{i} \theta_{i}^{(n-1)}$, it is not difficult to get

$$
\left|\delta^{(n)}\right| \leq \gamma h \sum_{i=1}^{j}\left|R_{i}\right|=\gamma h \sum_{i=1}^{j}\left|c_{1}^{i}\right|\left|\theta_{i}^{(n-1)}\right|,
$$

and thereby

$$
\left\|\delta^{(n)}\right\|_{\infty} \leq \gamma\left\|C_{1}\right\|_{\infty}\left\|\theta^{(n-1)}\right\|_{\infty}=\rho_{1}\left\|\theta^{(n-1)}\right\|_{\infty} .
$$

In similar manner, from (24), (26) and $F_{i}=c_{2}^{i} \delta_{i}^{(n)}$ we also obtain 


$$
\left\|\theta^{(n)}\right\|_{\infty} \leq 4 \alpha_{2}^{-1}\left\|c_{2}\right\|_{\infty},\left\|\delta^{(n)}\right\|_{\infty}=\rho_{2}\left\|\delta^{(n)}\right\|_{\infty} .
$$

Hence,

$$
\left\|\theta^{(n)}\right\|_{\infty} \leq \rho^{n-1}\left\|\theta^{(1)}\right\|_{\infty},\left\|\delta^{(n)}\right\|_{\infty} \leq \rho^{n-2} \rho_{1}\left\|\theta^{(1)}\right\|_{\infty} .
$$

The limit case for $n \rightarrow \infty$ leads to $\delta^{(n)}, \theta^{(n)} \rightarrow 0$. Hence the sequences $\left\{U_{i}\right\},\left\{V_{i}\right\}$ are Cauchy sequences and convergent:

$$
\lim _{n \rightarrow \infty} U_{i}^{(n)}=U_{i}, \lim _{n \rightarrow \infty} V_{i}^{(n)}=V_{i} .
$$

The limit functions $U_{i}, V_{i}$ will be solution of scheme (13)-(16).

Now we prove (21) and (22). We have

$$
\begin{aligned}
& \left\|U^{(n+m)}-U^{(n)}\right\|_{\infty} \\
& \leq\left\|U^{(n+m)}-U^{(n+m-1)}\right\|_{\infty}+\cdots+\left\|U^{(n+1)}-U^{(n)}\right\|_{\infty} \\
& \leq \rho_{1}\left(\rho^{n+m-1}+\rho^{n+m-2}+\rho^{n-1}\right)\left\|V^{(1)}-V^{(0)}\right\|_{\infty} \\
& =\rho_{1} \rho^{n-1}\left(\rho^{m-1}+\rho^{m-2}+\cdots+1\right)\left\|V^{(1)}-V^{(0)}\right\|_{\infty} \\
& =\rho_{1} \rho^{n-1} \frac{1-\rho^{m}}{1-\rho}\left\|V^{(1)}-V^{(0)}\right\|_{\infty} \\
& \leq \frac{\rho^{n-1}}{1-\rho}\left\|V^{(1)}-V^{(0)}\right\|_{\infty} .
\end{aligned}
$$

The limit case for $m \rightarrow \infty$ leads to (21). The inequality (22) is being proved analogously.

Lemma 2.3. The solution of the difference problem (13)-(16) satisfies

$$
\begin{aligned}
\|u\|_{\infty} \leq & \gamma(1-\rho)^{-1}\left\{\varepsilon \sigma_{*}^{(1)}\left|U_{x, 0}\right|+4 \alpha_{2}^{-1}\left\|c_{1}\right\|_{\infty}\right. \\
& \left.\times\left(\varepsilon \sigma_{*}^{(2)}\left|V_{x, 0}\right|+h \sum_{i=1}^{N-1}\left|f_{2}^{i}\right|\right)+h \sum_{i=1}^{N-1}\left|f_{1}^{i}\right|+\left|B_{2}\right|\right\}, \\
\|v\|_{\infty} \leq & 4 \alpha_{2}^{-1}(1-\rho)^{-1}\left\{\varepsilon \sigma_{*}^{(2)}\left|V_{x, 0}\right|+\gamma\left\|c_{2}\right\|_{\infty}\right. \\
& \left.\times\left(\varepsilon \sigma_{*}^{(1)}\left\|U_{x, 0}\right\|_{\infty}+h \sum_{i=1}^{N-1}\left|f_{1}^{i}\right|\right)+h \sum_{i=1}^{N-1}\left|f_{2}^{i}\right|+\left|B_{2}\right|\right\} .
\end{aligned}
$$

Proof. After setting $R_{i}=f_{1}^{i}+c_{1}^{i} V_{i}$ and $F_{i}=f_{2}^{i}+c_{2}^{i} U_{i}$ we will get

$$
\begin{aligned}
\|U\|_{\infty} \leq \gamma\left(\varepsilon \sigma_{*}^{1}\left|U_{x, 0}\right|+\left\|c_{1}\right\|_{\infty}\|V\|_{\infty}+h \sum_{i=1}^{N-1}\left|f_{1}^{i}\right|\right), \\
\|V\|_{\infty} \leq 4 \alpha_{2}^{-1}(1-\rho)^{-1}\left\{\varepsilon \sigma_{*}^{(2)}\left|V_{x, 0}\right|+\gamma\left\|c_{2}\right\|_{\infty}\right. \\
\left.\times\left(\varepsilon \sigma_{*}^{(1)}|| U_{x, 0} \|_{\infty}+h \sum_{i=1}^{N-1}\left|f_{1}^{i}\right|\right)+h \sum_{i=1}^{N-1}\left|f_{2}^{i}\right|+\left|B_{2}\right|\right\} .
\end{aligned}
$$

The using each of these into another immediatelleads to (27) and (28).

Lemma 2.4. For the truncation errors

$$
\begin{gathered}
R_{1}^{i}=f_{1}\left(x_{i}\right)-L_{1}^{h} u\left(x_{i}\right)+c_{1}\left(x_{i}\right) v\left(x_{i}\right), \\
R_{2}^{i}=f_{2}\left(x_{i}\right)-L_{2}^{h} v\left(x_{i}\right)+c_{2}\left(x_{i}\right) u\left(x_{i}\right), \\
r_{0}^{(1)}=\left(1+a_{1}(0) \frac{h}{\varepsilon} \sigma_{10}^{(2)}\right)^{-1}\left(\varepsilon^{-1} B_{1}-b_{1}(0) A_{1} \frac{h}{\varepsilon} \sigma_{10}^{(2)}\right. \\
\left.+\frac{h}{\varepsilon} \sigma_{10}^{(2)} f(0)\right)-U_{x, 0}, \\
r_{0}^{(2)}=\frac{A_{2}\left(1-\exp \left(-a_{2}(0) h / \varepsilon\right)\right)}{a_{2}(0) h}-V_{x, 0} \\
=\frac{A_{2}}{\varepsilon\left(1+a_{2}(0) \frac{h}{\varepsilon} \sigma_{20}^{(2)}\right)}-V_{x, 0},
\end{gathered}
$$

the following estimates hold

$$
\begin{aligned}
\left|R_{1}^{i}\right| \leq C h\left(1+h^{-1} \int_{x_{i-1}}^{x_{i+1}}\left|u^{\prime}(x)\right| \mathrm{d} x+h^{-1} \int_{x_{i-1}}^{x_{i+1}}\left|v^{\prime}(x)\right| \mathrm{d} x\right), \\
\left|R_{2}^{i}\right| \leq C h\left(1+h^{-1} \int_{x_{i-1}}^{x_{i+1}}\left(\left|v^{\prime}(x)\right|+\left|v^{\prime}(x)\right|\right)\right) \mathrm{d} x, \\
\left|r_{0}^{(1)}\right| \leq \frac{C h}{\varepsilon\left(1+a_{1}(0) \sigma_{10}^{(2)} h / \varepsilon\right)} \\
\times\left(1+h \int_{x_{0}}^{x_{1}}\left|u^{\prime}(x)\right| \mathrm{d} x+\int_{x_{0}}^{x_{1}}\left|v^{\prime}(x)\right| \mathrm{d} x\right) \\
\left|r_{0}^{(2)}\right| \leq \frac{C h}{\varepsilon\left(1+a_{2}(0) \sigma_{20}^{(2)} h / \varepsilon\right)} \\
\times\left(1+\int_{x_{0}}^{x_{1}}\left|v^{\prime}(x)\right| \mathrm{d} x+\int_{x_{0}}^{x_{1}}\left|u^{\prime}(x)\right| \mathrm{d} x\right) .
\end{aligned}
$$

Proof. We may write

$$
\begin{aligned}
R_{1}^{i}= & h^{-1} \int_{x_{i-1}}^{x_{i+1}}\left(a_{1}(x)-a_{1}\left(x_{i}\right)\right) u^{\prime}(x) \varphi_{i}(x) \mathrm{d} x \\
& +h^{-1} \int_{x_{i+1}}^{x_{i-1}}\left(b_{1}(x) u(x)-b\left(x_{i}\right) u\left(x_{i}\right)\right) \varphi_{i 1}(x) \mathrm{d} x \\
& +h^{-1} \int_{x_{i-1}}^{x_{i+1}}\left(f_{1}\left(x_{i}\right)-f(x)\right) \varphi_{i 1}(x) \mathrm{d} x \\
& +h^{-1} \int_{x_{i-1}}^{x_{i+1}}\left(c_{1}\left(x_{i}\right) v\left(x_{i}\right)-c_{1}(x) v(x)\right) \varphi_{i 1}(x) \mathrm{d} x,
\end{aligned}
$$




$$
\begin{aligned}
R_{2}^{i}= & h^{-1} \int_{x_{i-1}}^{x_{i+1}}\left(a_{2}(x)-a_{2}\left(x_{i}\right)\right) v^{\prime}(x) \varphi_{i 2}(x) \mathrm{d} x \\
& -h^{-1} \int_{x_{i-1}}^{x_{i+1}}\left(b_{2}(x) v(x)-b_{2}\left(x_{i}\right) v\left(x_{i}\right)\right) \varphi_{i 2}(x) \mathrm{d} x \\
& +h^{-1} \int_{x_{i-1}}^{x_{i+1}}\left(f_{2}\left(x_{i}\right)-f_{2}(x)\right) \varphi_{i 2}(x) \mathrm{d} x \\
& +h^{-1} \int_{x_{i-1}}^{x_{i+1}}\left(c_{2}\left(x_{i}\right) u\left(x_{i}\right)-c_{2}(x) u(x)\right) \varphi_{i 2}(x) \mathrm{d} x
\end{aligned}
$$

$$
\begin{aligned}
r_{0}^{(1)}= & \frac{1}{\varepsilon\left(1+a_{1}(0) \frac{h}{\varepsilon} \sigma_{10}^{(2)}\right)} \\
& \times\left\{\int_{x_{0}}^{x_{1}}\left(b_{1}(x) u(x)-b_{1}(0) u(0)\right) \varphi_{01}^{(2)}(x) \mathrm{d} x\right. \\
& +\int_{x_{0}}^{x_{1}}\left(a_{1}(x)-a_{1}(0)\right) u^{\prime}(x) \varphi_{01}^{(2)}(x) \mathrm{d} x \\
& -\int_{x_{0}}^{x_{1}}\left(f_{1}(x)-f_{1}(0)\right) \varphi_{01}^{(2)}(x) \mathrm{d} x \\
& \left.-\int_{x_{0}}^{x_{1}} c_{1}(x) v(x) \varphi_{01}^{(2)}(x) \mathrm{d} x\right\},
\end{aligned}
$$$$
r_{0}^{(2)}=\frac{1}{\varepsilon\left(1+a_{2}(0) \frac{h}{\varepsilon} \sigma_{20}^{(2)}\right)}
$$$$
\times\left\{\int_{x_{0}}^{x_{1}}\left(a_{2}(x)-a_{2}(0)\right) v^{\prime}(x) \varphi_{02}^{(2)}(x) \mathrm{d} x\right.
$$$$
-\int_{x_{0}}^{x_{1}} b_{2}(x) v(x) \varphi_{02}^{(2)}(x) \mathrm{d} x-\int_{x_{0}}^{x_{1}} f_{2}(x) \varphi_{02}^{(2)}(x) \mathrm{d} x
$$$$
\left.-\int_{x_{0}}^{x_{1}} c_{2}(x) u(x) \varphi_{02}^{(2)}(x) \mathrm{d} x\right\}
$$

where

$$
\varphi_{i k}= \begin{cases}\varphi_{i k}^{(1)}(x)=\frac{\mathrm{e}^{a_{i k}\left(x-x_{i-1}\right) / \varepsilon}-1}{\mathrm{e}^{a_{i k} h / \varepsilon}-1}, & x_{i-1}<x<x_{i}, \\ \varphi_{i k}^{(2)}(x)=\frac{1-\mathrm{e}^{-a_{i k}\left(x_{i-1}-x\right) / \varepsilon}}{1-\mathrm{e}^{a_{i k} h / \varepsilon}}, & x_{i}<x<x_{i+1}, \\ 0, & x \notin\left(x_{i-1}, x_{i+1}\right) .\end{cases}
$$

The relations (33)-(36) leads immediately to (29)-(32).

Theorem 2.3. Let

$a_{k}(x), b_{k}(x), c_{k}(x), f_{k}(x) \in C[0,1]$. Then the solution of the difference problem (13)-(16) converges uniformly in $\varepsilon$ to the solution of (1)-(4) with rate

Proof. Let

$$
z_{1 i}=U_{i}-u\left(x_{i}\right), z_{2 i}=V_{i}-v\left(x_{i}\right) .
$$

Then for the errors of the approximate solution $z_{k i}(k=1,2 ; i=\overline{0, N})$ we have

$$
\begin{aligned}
& L_{1}^{h} z_{1 i}=c_{1}^{i} z_{2 i}+R_{1}^{i},(i=\overline{1, N-1}), \\
& L_{2}^{h} z_{2 i}=c_{2}^{i} z_{1 i}+R_{2}^{i}, \\
& z_{10}=0, z_{1 x, 0}=r_{0}^{(1)}, \\
& z_{2 x, 0}=r_{0}^{(2)}, z_{2 N}=0,
\end{aligned}
$$

where $R_{1}^{i}, R_{2}^{i}, r_{0}^{(1)}, r_{0}^{(2)}$ are approximating errors from Lemma 2.4. Using Lemma 2.3 we obtain

$$
\begin{aligned}
& \left\|z_{1}\right\|_{\infty},\left\|z_{2}\right\|_{\infty} \\
& \leq C\left\{\varepsilon \sigma_{*}^{(1)}\left|r_{0}^{(1)}\right|+\varepsilon \sigma_{*}^{(2)}\left|r_{0}^{(2)}\right|+h \sum_{i=1}^{N-1}\left|f_{2}^{i}\right|+h \sum_{i=1}^{N-1}\left|f_{1}^{i}\right|\right\} .
\end{aligned}
$$

By virtue that of (29)-(32) all terms in right-hand side of this inequality have the rate $O(h)$ and hence the proof follows immediately

\section{REFERENCES}

[1] A. H. Nayfeh, "Introductions to Pertubation Techniques," Wiley, New York, 1993.

[2] E. R. Doolan, J. J. H. Miller and W. H. A. Schilders, "Uniform Numerical Methods for Problems with Initial and Boundary Layers,” Boole Press, Dublin, 1980.

[3] I. G. Amiraliyeva, "Uniform Difference Scheme on the Singulary Pertubed System," Applied Mathematics, Vol. 3, 2012, pp. 1029-1035. http://dx.doi.org/10.4236/am.2012.39152

[4] P. A. Farrell, A. F. Hegarty, J. J. H. Miller, E. O’Riordan and G. I. Shishkin, "Robust Computational Techniques for Boundary Layers,” Chapman-Hall/CRC, New York, 2000.

[5] G. M. Amiraliyev and H. Duru, "A Uniformly Convergent Finite Difference Method for a Initial Value Problem," Applied Mathematics and Mechanics, Vol. 20, No. 4, 1999, pp. 363-370. http://dx.doi.org/10.1007/BF02458564

[6] G. M. Amiraliyev, "The Convergence of a Finite Difference Method on Layeradapted Mesh for a Singulary Pertubed System," Applied Mathematics and Computation, Vol. 162, No. 3, 2005, pp. 1023-1034. http://dx.doi.org/10.1016/j.amc.2004.01.015

[7] H. G. Roos, M. Stynes and L. Tobiska, "Numerical Methods for Singulary Pertubed Differential Equations, Convection Diffusion and Flow Problems,” Springer-Verlag, Berlin, 1996. http://dx.doi.org/10.1007/978-3-662-03206-0 
[8] R. E. O’Malley, "Singular Pertubations Methods for Ordinary Differential Equations,” Springer Verlag, New York, 1991. http://dx.doi.org/10.1007/978-1-4612-0977-5

[9] S. Natesan and B. S. Deb, “A Robust Computational Method for Singularly Pertubed Coupled System of Reaction-Diffusion Boundary-value Problems,” Applied Mathematics and Computation, Vol. 188, No. 1, 2007, pp. 353-364. http://dx.doi.org/10.1016/j.amc.2006.09.120

[10] S. Hemavathi, T. Bhuvaneswari, S. Valarmathi and J. J. H. Miller, "A Parameter Uniform Numerical Method for a
System of Singularly Pertubed Ordinary Differential Equations,” Applied Mathematics and Computation, Vol. 191, No. 1, 2007, pp. 1-11. http://dx.doi.org/10.1016/j.amc.2006.05.218

[11] Z. D. Cen, A. M. Xu and A. B. Le, "A Second-Order Hybrid Finite Difference Scheme for a System of Singularly Pertubed Initial Value Problems,” Journal of Computational and Applied Mathematics, Vol. 234, No. 12, 2010, pp. 3445-3457.

http://dx.doi.org/10.1016/j.cam.2010.05.006 